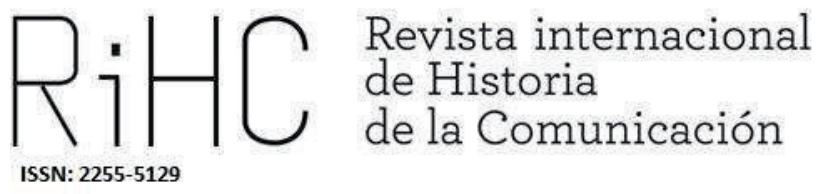

\title{
ENTRANDO EN LOS NOVENTA: FEMINISMO DE ESTADO E IMAGEN DE LA MUJER TRABAJADORA EN LAS FICCIONES DE TVE
}

Into the nineties. State feminism and the representation of working-class women in TVE Fictions

DOI: http://dx.doi.org/10.12795/RiCH.2021.i16.10

Recibido: 17-4-2021

Aceptado: $10-6-2021$

Publicado: 30-6-2021

Javier Jurado

Université de Lille, Francia

juradog@gmail.com

ORCID (D) https://orcid.org/0000-0002-7025-9427 
Resumen: En este artículo nos adentramos en las representaciones que TVE difunde de la mujer trabajadora en las series de ficción durante la tercera legislatura de Felipe González. Son los años en los que el Instituto de la Mujer establece el primer Plan para la Igualdad de Oportunidades de las Mujeres (PIOM, 1988-1990) que favorece la integración masiva de las mujeres en el mercado laboral remunerado, esto es, a su "profesionalización". Esta imagen se transmite en las series en la que la caracterización y las narrativas se presentan bajo la óptica patriarcal respecto a la imagen de la mujer. Es más, las mujeres de clase trabajadora están sistemáticamente invisibilizadas y/o minusvaloradas a través de bromas sexistas, violencia verbal...

Palabras clave: Mujeres de clase trabajadora. Televisión pública. Clasismo. Socioliberalismo

\begin{abstract}
In this article I will explore the representation of women according to their social status in television series shown during the period 1989-1993 on Spanish public television (TVE). While there are a significant number of young active women occupying key roles in series made during this period - a move influenced by the Equal Opportunity Plan for Women (PIOM) from the ruling Socialist Party and the Women's Institute - their characterisation and narrative function remain heavily attached to a patriarchal view of women. Furthermore, working-class women are systematically under-represented and/or undervalued through sexist jokes, verbal violence and mockery towards housewives.
\end{abstract}

Keywords: Working-class women. Public broadcasting. Social bias. Socio-liberalism

\title{
1 Introducción
}

En marzo de 1990, TVE proyectó por primera vez el drama más caro de su historia: La forja de un rebelde (Mario Camus, 1990). Se basa en la autobiografía de Julián Barea, desde sus primeros años en el barrio obrero de Lavapiés en Madrid hasta su papel como jefe de la censura republicana durante la Guerra Civil. Es una historia de sufrimiento, pero también de ascenso social, un argumento caro a los dramas históricos de TVE en los años 80 y 90, haciéndose eco del programa político y económico del PSOE de Felipe González. Este tipo de relatos son protagonizados en general por hombres que consiguen ascender en la escala social gracias a su esfuerzo individual (Jurado, 2017). Frente a este predominio de personajes masculinos, nos parece relevante interpretar los tipos de representaciones que las series de esta época ofrecen de las mujeres, prestando especial atención a las diferencias de tratamiento según su clase social.

Desde la revolución industrial, la división de los espacios públicos y privados y la asignación de roles productivos y reproductivos han condenado a la invisibilidad y gratuidad las actividades tradicionalmente realizadas por las mujeres, y han llevado a considerarlas como tareas excluidas del mercado laboral (Federici, 2012: 18) (Fraser, 2016: 102). Esta distinción, hoy hegemónica en las sociedades capitalistas, entre trabajo 
remunerado y actividades no remuneradas excluidas del mercado, crea representaciones sociales bien ancladas en las mentalidades (Ruido, 2007). Esta concepción ha sido ciertamente cuestionada por diversas autoras feministas desde los años 70, entre ellas Silvia Federici, pero no es menos cierto que la mayoría de los estudios que se dirigen a las producciones audiovisuales desde los años 90 (McRobbie, 2009: 33), incluidos los estudios de género, rara vez se centran en la importancia del mundo del trabajo en la construcción del género femenino (Lacalle \& Gómez, 2016).

Pero si el mundo del trabajo nos interesa en nuestro análisis, es también porque, en nuestras sociedades postindustriales, el trabajo desempeña paradójicamente un papel crucial en la definición de la propia identidad. En una época también llamada "postsocialista" por Nancy Fraser (1995), la política del reconocimiento ha sustituido a la de redistribución característica de la posguerra, convirtiendo la profesión en una apuesta fundamental en esta economía del valor individual. Así, el discurso del feminismo liberal concede especial importancia al acceso de las jóvenes al mercado laboral cualificado, así como al mercado de consumo (McRobbie, 2009: 9). Así, tenemos un modelo de mujer, promovido por las instituciones públicas y reforzado por los discursos del Instituto de la Mujer y del primer PIOM, que se traduce, como demostraremos, en la preponderancia de mujeres jóvenes, activas, urbanas y de clase media en las ficciones de TVE.

El año 1989 marca el fin del monopolio de la radiodifusión a nivel nacional de TVE, con la creación de la FORTA ${ }^{1}$ y el desarrollo de las cadenas privadas. En este marco, la televisión pública sigue un modelo de programación que prioriza la calidad, un modelo importado por Pilar Miró durante su etapa en la Dirección General de Cine y que pronto abandonó en favor de cálculos económicos más o menos juiciosos (Duran-Froix, 2009: 1-5). Fueron, además, los años en los que el Instituto de la Mujer puso en marcha su primer Plan de Igualdad de Oportunidades para la Mujer (PIOM, 1988-1990), promoviendo la profesionalización y la incorporación masiva de la mujer al mercado laboral remunerado.

El interés por la representación de las mujeres en estas series es tanto más importante cuanto que ellas son su principal público (AAVV, 2004: 25-26). Las políticas públicas del PSOE y las iniciativas del Instituto de la Mujer, especialmente durante los años en que se puso en marcha el Plan de lgualdad de Oportunidades, trataron de crear las condiciones para la incorporación al mundo laboral de las mujeres. Los dramas de TVE también van en esta dirección, y la gran mayoría de los personajes femeninos de las series que analizaremos -al menos en cuanto a las protagonistas, es decir, las que desempeñan papeles importantes en las tramas- son jóvenes trabajadoras de clase media alta. ¿Qué espacio queda pues para las categorías sociales más modestas?

\footnotetext{
${ }^{1}$ Federación de Organismos de Radio y Televisión Autonómicos, asociación fundada en 1989.
} 


\subsection{Metodología}

En este artículo interpretaremos los tipos de representación que las series de la TVE han hecho de las mujeres, en relación con su situación profesional y económica, en un momento que nos parece especialmente significativo desde el punto de vista institucional, político y cultural. Nos centraremos en la tercera legislatura del PSOE (1989-1993), un periodo en el que el Estado perdía el monopolio de la producción televisiva y en el que las lógicas comerciales empezaban a hacerse evidentes. Este, nos parece, un momento privilegiado para estudiar tanto las estrategias del gobierno y de los profesionales de la televisión, que pretenden fidelizar a la audiencia frente a la creciente competencia, como el modelo de sociedad que promueven estos actores.

De este modo pretendemos responder a las siguientes preguntas ¿Qué representaciones de la mujer ofrecía la televisión pública española a principios de los 90? ¿Hasta qué punto la clase social y la agenda feminista institucional desempeñaron un papel en su desarrollo? Y, por último, ¿de qué manera podrían haberse utilizado los dramas televisivos para difundir determinado modelo? Para ello analizamos las catorce series producidas por TVE y emitidas entre 1989 y 1992, de las que la mitad están ambientadas en el pasado y la otra mitad en la época más contemporánea. Éstas son: Brigada Central (1989), Pedro I el Cruel (1989), Delirios de amor (1989), El olivar de Atocha (1989), Miguel Servet (1989), La forja de un rebelde (1990), La mujer de tu vida (1990), Eva y Adán. Agencia Matrimonial (1990), Chicas de hoy en día (1991), Historias del otro lado (1991), Los jinetes del Alba (1991), Una hija más (1991), El Quijote (1992) y Hasta luego cocodrilo (1992). Este periodo nos parece particularmente relevante por la competencia a la que se sometía TVE por parte de los nuevos competidores autonómicos y privados, y, como veremos, por la importancia de la publicidad en la programación televisiva española.

Para responder a las preguntas planteadas al principio del artículo abordamos estas ficciones como productos industriales, culturales e históricos. Dado que fueron los años de transición del modelo "institucional" al modelo "comercial" estimulado por la aparición de canales privados altamente capitalizados (Arranz, 2010: 43), consideramos necesario realizar un análisis de las lógicas económicas e industriales que operan en la producción de seriales (Barthes, 2011: 49) en relación con la distribución de género de las categorías profesionales. Por tanto, un análisis de la producción se impone, mediante la contextualización que permite comprender tanto el dispositivo enonciativo, como la doxa, esto es, el conjunto de discursos implícitos y explícitos que permiten la identificación del espectador con la cosmovisión compartida en un determinado contexto social (Sepulchre, 2017: 119-120).

Este análisis tiene lugar en la primare parte del artículo en la que nos detenemos en los condicionantes industriales y económicos del medio televisivo. La fidelidad de la audiencia, el peso de la publicidad y la rentabilidad de las producciones son factores que 
condicionan el contenido de las series, su ubicación en la parrilla de programación y su durabilidad. Pero las ficciones audiovisuales también participan en la creación de imaginarios colectivos, en la construcción de representaciones de la realidad (Macé, 2000: 249) así como en la producción de subjetividad (De Lauretis, 1984: 34), aspectos todos ellos que también son esenciales para analizar la dimensión cultural de las series y los modelos de sociedad que transmiten como demostramos en la segunda parte del trabajo.

En efecto, compartimos la idea de Sabine Chalvon-Demersay (2015: 37) que los personajes sirven de intermediarios entre los espectadores y la ficción y que, estudiando su lenguaje, puntos de vista, poniéndolos en perspectiva, documentándolos a la vez desde el punto de vista técnico y político, tomando en serio las ideas que desarrollan, explicitar sus contradicciones... podemos dilucidar su rol de actor social en el mundo que se proyectan. La transposición del mundo de ficción al de la realidad de los espectadores es mediado aquí por la filiación genérica de las series que, como veremos, derivan de una cierta voluntad de legitimación. Es por ello que la identificación y clasificación de los personajes (método cuantitativo) así como el análisis fílmico y narratológico de la representación de género (cualitativo) se complementa aquí con la contextualización política, industrial y económica que nos permite dilucidar las representaciones vehiculadas por estas ficciones.

\section{El precio de la integración. Estado, industria y publicidad}

El final de la década de 1980 marcó la consolidación del PSOE como partido hegemónico del centro-izquierda español. Tras las movilizaciones del final del franquismo y la Transición, el partido de Felipe González llevó a cabo una política de consenso y modernización que, en 1986, culminaría con la integración del país en la CEE y la OTAN. Las políticas socialistas no encontraron una oposición significativa en la derecha: a pesar de su avance frente a la UCD de Adolfo Suárez, Alianza Popular no consiguió constituir una alternativa sólida. El Ejército había aceptado el juego democrático -y un ministro civil- tras el golpe de Estado de 1981 y la Iglesia, que había perdido el monopolio de la educación, tuvo que conformarse con las concesiones otorgadas por el gobierno. Dentro de la izquierda parlamentaria, el Partido Comunista se diluía en una nueva coalición (Izquierda Unida) que, por el momento, era incapaz de federar de manera masiva el descontento con las políticas liberales del gobierno. Aparte de las actividades de ETA, las únicas fuerzas que aún podían constituir un contrapeso eran los movimientos sociales y culturales, muy activos durante las décadas de 1960 y 1970. 
Estudiantes, objetores de conciencia, movimientos anticoloniales, homosexuales, feministas y trabajadores pudieron coordinar sus luchas gracias a la participación de artistas, emisoras de radio piratas, fanzines y, por supuesto, películas y documentales. Este movimiento fue conocido bajo el apelativo de contracultura, núcleo de iniciativas de carácter utópico y proactivo. Pretendía provocar un cambio cultural en respuesta a las aspiraciones de liberación, concibiendo la modernidad como la superación de los anclajes tradicionales. Sin embargo, el desgaste provocado por la política de consenso de los Pactos de la Moncloa, los estragos causados por la llegada masiva de la heroína en los años 80 y la comercialización de la contracultura, cuyo icono es la Movida Madrileña, llevaron a un estado de depresión social conocido como desencanto.

Tras las victorias electorales socialistas, primero a nivel local y regional, y luego en las elecciones legislativas de 1982, varios miembros de estos colectivos comenzaron entonces a integrarse en las estructuras de la administración y del partido para asegurar la supervivencia y la financiación de sus movimientos. En el caso de varios integrantes de la lucha feminista, con la creación del Instituto de la mujer y la institucionalización de sus reivindicaciones, llevadas ahora por un "feminismo de Estado" (Valiente, 1994), cuyo acceso a las responsabilidades políticas alejaba de las aspiraciones más o menos revolucionarias.

La integración en los organismos públicos implicaba de hecho la aceptación de la política de consenso establecida por un PSOE que, desde la llegada de Felipe González a su cabeza, era una organización disciplinada y centralizada (Rodriguez López, 2015, p. 276). La modernidad para el partido gobernante significaba gestión tecnocrática y consenso apolítico siguiendo el ejemplo de la socialdemocracia alemana y las teorías de Ulrich Beck -más tarde aplicadas en el Reino Unido con la Tercera Vía laborista- en un consenso internacional de centro-izquierda (Giddens, 1998) que debía combatir la revolución conservadora en su propio terreno. Esta política de consenso fue considerada por sectores más contestatarios como una renuncia tras el auge y la coordinación progresiva de las luchas sociales durante los años 60 y 70 . Una retirada que implica una "desarticulación" de las luchas (McRobbie, 2009: 24-53) y una desradicalización en favor de los cálculos electorales y la integración en las políticas culturales europeas.

En el contexto de las luchas feministas, esta integración en el aparato estatal era en cierto modo necesaria para hacer avanzar las políticas públicas que, bajo los gobiernos de UCD, sólo evolucionaban gracias a la presión social. Un proceso sin embargo no exento de problemas y contradicciones, como la dificultad de exigir cambios a las administraciones a la vez que se forma parte de ellas (Walby, 2005). Como resultado, se fueron desinflando reivindicaciones sociales a cambio de compromisos concretos de los poderes públicos. Uno de esos compromisos fue el Plan de Igualdad de Oportunidades para la Mujer de 1988, que incluía la sensibilización en los ámbitos educativo y cultural. Los cuatro objetivos del plan, recogidos en los textos del Ministerio de Cultura, son 
combatir los estereotipos sexistas, fomentar un cambio de actitud de los profesionales de la educación, garantizar la igualdad de oportunidades para las jóvenes y adaptar la formación permanente a las necesidades específicas de las mujeres (AAVV, 1987). Otro ejemplo es la Ley General de Publicidad, que pretendía mejorar la imagen de la mujer en los medios de comunicación.

\subsection{La configuración de un nuevo modelo industrial}

La televisión siendo un medio de flujo (Williams, 1990: 86) está sujeta a las condiciones de producción industrial y de emisión, en la medida en que debe ofrecer un contenido continuo que mantenga a los espectadores frente a la pantalla. Como sostiene Román Gubern (2000: 32), esta lógica económica, que proviene del fordismo y el taylorismo, se aplica a la industria audiovisual en el caso de la serialidad televisiva. Su carácter redundante y familiar y sus mínimas variaciones se convierten en elementos esenciales. Sin embargo, es necesario recordar que la producción serial también estuvo muy presente en el origen del cine, y que el deseo de fidelizar al público, en las salas de cine o frente a la pequeña pantalla, responde a "prácticas ligadas a la industrialización del campo artístico y cultural" (Saute-Requin, 2019: 6).

Las series son uno de los productos estrella de la televisión, con un importante índice de seguimiento y demanda, y por tanto uno de los programas más rentables para las cadenas. Por lo tanto, su objetivo es atraer y retener al público. Para ello, la mayoría de ellos abordan temas familiares, centrados en la vida cotidiana buscando la cercanía con la población (Eskenazi, 2017: 36-37). Tras los índices de audiencia se encuentra la cuestión central de la rentabilidad que en el caso de la televisión española la ha hecho depender de los anunciantes desde 1965, lo que provocaría que poco a poco la dictadura perdiera el control sobre los contenidos emitidos (Jurado, 2021: 230). La importancia de la publicidad en el desarrollo de los programas y de la parrilla de programación se acentuó en estos años 90, cuando TVE empezó a darse cuenta de que la pérdida de audiencia podía implicar una merma de ingresos publicitarios (Moreno Díaz \& Medina, 2017: 68). Así, teniendo en cuenta el peso de la publicidad en la financiación de la televisión pública en los años ochenta y noventa, no parece abusivo afirmar que las series de televisión son objeto de un doble proceso de consumo: de la propia ficción y de la publicidad, en forma de anuncios o product placements. En resumen, y citando a Beverley Skeggs (2004: 97):

Aquellos que disponen de un poder simbólico crean representaciones que circulan a través de varios sistemas de distribución por varias razones: para vender espacio televisivo a anunciantes y por lo tanto usar representaciones para ganar altos índices de audiencia; para generar "verdad" y "autenticidad" sobre el otro; para 
identificar y contener problemas sociales; 0 , a veces, simplemente rellener espacio televisivo. Sin embargo, estos intereses no son siempre evidentes; varias de las motivaciones que están detrás de estas representaciones no tienen nada que ver con cierta intencionalidad, sino más bien con la ignorancia de los productores y su falta de conocimiento de las culturas que representan.

Las series producidas por TVE permiten estudiar el modo en que se plasman los discursos públicos sobre el acceso de las mujeres al mundo del trabajo, así como la imagen que los poderes públicos proponen de ellas, especialmente durante estos últimos años de monopolio catódico. Es importante tener en cuenta que todas las series analizadas, aunque sean desarrolladas por productoras privadas, cuentan en sus filas con delegados del organismo público que supervisa estas ficciones -en el caso de Brigada Central (1989), su guionista, el novelista Juan Madrid, denunció varias situaciones de injerencia política a las que se refiere como evidente censura por parte del gobierno ${ }^{2}-$. Otro aspecto que a menudo se ignora en el estudio de las series es su ubicación en la parrilla de programación que nos indica el tipo de público al que se dirige, pero también la importancia que se da a cada una de estas producciones. Por último, es pertinente señalar que, en nuestro corpus, son mayoría los hombres que dirigen (sólo 2 mujeres para las 14 series, incluida una codirectora) y escriben los guiones (15 mujeres de un total de 48 guionistas para los 157 episodios analizados) y que en varios casos en los que las mujeres realizan esta labor de producción, sus nombres no figuran en los créditos.

Esta infrarrepresentación de las mujeres en puestos clave de la creación audiovisual afecta a los discursos, representaciones e imaginarios del mundo del trabajo femenino que vemos en la pantalla. Sin embargo, hay que recordar que incluso la progresiva feminización de este medio, hasta la cabecera de Televisión Española, no excluye la interiorización de la mirada masculina según la teoría clásica de Mulvey (1975). A esta mirada masculina se añade un evidente sesgo de clase que condiciona las representaciones de las mujeres en los productos audiovisuales y sobrevalora a las mujeres de estatus social privilegiado en relación con las clases subalternas. En efecto, la implantación por parte del PSOE de un feminismo de Estado (que la búsqueda de consenso vacía, en parte, de contenido) y las recomendaciones del Instituto de la Mujer (administrado por gestores progresivamente despolitizados), suponen un olvido de las reivindicaciones feministas radicales y populares de los años 70 . Además, la contratación de profesionales de los medios de comunicación, así como de representantes políticos e institucionales - en su mayoría procedentes de las clases acomodadas- crea una permeabilidad al discurso neoliberal, que rara vez es reconocida por los interesados.

2 "La Transición fue una gran mentira pactada" Público, 31/10/2008 https://www.publico.es/actualidad/transicion-gran-mentira-pactada.html (último acceso 07/06/2021) 


\subsection{Consumo(s)}

Ya hemos estudiado el impacto del discurso tecnocrático en los medios de comunicación a partir de los años 60 (Jurado, 2021: 229 y ss.). Así, se recurre a la figura del hombre de negocios como proveedor del sustento familiar. La transición democrática y las luchas feministas impulsaron un cambio cultural que modificó radicalmente la representación de las mujeres en los medios de comunicación: visibilidad pública, acceso al mercado laboral, independencia económica, etc. Como consecuencia, los discursos de género que transmiten las producciones audiovisuales también se han visto afectados. Las mujeres se presentan ahora como jóvenes activas, implicadas en las dinámicas de consumo y de producción de valor de mercado. En este sentido, responden a una sensibilidad postfeminista basada en una construcción neoliberal en la que la persona, concebida como proyecto, necesita ser transformada continuamente, la mayoría de las veces a través de modos de consumo (Riley, Evans, \& Mackiewicz, 2016).

La sobrerrepresentación de las mujeres en las profesiones cualificadas es un marcador que se repite desde los años 70 en la ficción estadounidense (Lacalle \& Gómez, 2016: 60-61) y que encontramos en las series estudiadas. Entre los personajes principales hay dos policías, una maestra de escuela, una secretaria, una psicóloga, dos actrices, una violinista, y sólo una criada y una camarera de piso. Esta distribución refleja los nuevos roles sociales de la mujer: autónoma, económicamente independiente y de clase media. Tienen entre 25 y 40 años, en su mayoría solteras y constituyen un objeto privilegiado para la escopofilia del público. Las protagonistas femeninas de las series posmodernas ya no suelen estar sujetas al ciclo tradicional de matrimonio, procreación y envejecimiento en el hogar. Ahora se ponen en escena, se hacen visibles como mujeres activas e independientes.

Si la televisión pública difunde así un nuevo modelo femenino, su público potencial $-y$ también el que busca- es, sin embargo, mucho más amplio. La lógica económica que acabamos de describir nos informa de la centralidad de las cuestiones publicitarias y de su influencia en el discurso transmitido por la esfera audiovisual y las opciones de programación. Así, los anunciantes buscan despertar y mantener los deseos de consumo de los espectadores, creando en su mayoría la ilusión de un mundo urbano y cosmopolita, marcado por la abundancia. El problema es que las mujeres que componen la audiencia de estos programas rara vez forman parte de este mundo laboral urbano: en su mayoría son amas de casa o tienen trabajos menores y, en cualquier caso, siguen ganando menos dinero que sus homólogos masculinos, lo que las hace más dependientes de los servicios públicos. Así, las mujeres que constituyen la audiencia mayoritaria de estas series están claramente infrarrepresentadas.

Al mostrar el estilo de vida de las clases medias a un público que procede en gran medida de las clases trabajadoras, se disocian los dos aspectos en los que se basa la economía audiovisual: el consumo de los productos vendidos por los anunciantes, que concierne 
al público con recursos económicos suficientes -o con acceso al crédito- y el consumo de los propios programas, que concierne a una parte mucho más amplia de la población. La posibilidad de que el público se identifique con los protagonistas de los productos audiovisuales estudiados desempeña un papel fundamental en este proceso de doble consumo. Dado que los personajes y grupos sociales representados en estas series son en su mayoría de clases acomodadas y urbanas, la pertenencia social se convierte en un factor importante para identificarse con los roles que estas ficciones retratan. Sin embargo, esto no significa una aceptación total o un rechazo consciente por parte de quienes consumen estas ficciones (De Lauretis, 1984: 37).

\section{Las mujeres en la pantalla}

A día de hoy la idea del progreso económico, el acceso a la propiedad y el consumo que permitiría la movilidad social ascendente prometida por la revolución conservadora y socioliberal es menos evidente de lo que los discursos podrían sugerir. El auge del sector de los servicios no ha provocado un aumento masivo de los empleos de alto valor añadido, al menos en los países de la Unión Europea, y menos aún en los países del este y del sur del continente. Sin embargo, el consumo es necesario para mantener este sistema económico, que también influye en el modelo de feminidad en las sociedades capitalistas actuales.

Como anunciamos en el apartado metodológico, nos encontramos con dos tipos de producciones seriadas: por un lado, las series históricas, que tienen sus raíces en un pasado más o menos lejano, y por otro, las series cuya trama se desarrolla en el mundo contemporáneo. La ficción histórica ha desempeñado durante mucho tiempo un papel fundamental en el panorama audiovisual español. La narración de la historia ha sido una herramienta privilegiada para legitimar el poder. Durante el periodo que va de 1989 a 1993, el peso de las series históricas producidas por TVE sigue siendo considerable: representa casi la mitad del total con seis de las catorce producciones, a las que se suma una séptima, Hasta luego cocodrilo, que es un retrato generacional de la época construido a partir de flashbacks que transportan a la audiencia durante los años del final de la dictadura y la Transición. Este predominio está en consonancia con la política cultural del gobierno socialista y su proyecto de nacionalización liberal que procede a revisar los discursos históricos del franquismo, desde un ángulo cada vez más despolitizado (Quaggio, 2013: 460). 


\subsection{El progreso social, una cuestión de género}

Una de las series históricas más caras de este periodo es Los jinetes del alba (Vicente Aranda, 1990). Es la única producción de nuestro corpus cuya trama se centra en la vida de una mujer, y la única que muestra su ascenso social. La ficción, inspirada en la novela de Jesús Fernández Santos (1984), está ambientada en el primer tercio del siglo XX y nos cuenta el recorrido biográfico de la protagonista. Marian (Victoria Abril), hija de una señora de la limpieza en un balneario de Asturias, se presenta a través de una voz en off en la segunda secuencia del primer episodio. En un lujoso comedor, la joven entra con una bandeja de fruta siguiendo a dos sirvientes. Tras un plano de seguimiento desde la puerta del complejo hasta la habitación en la que se encuentra, la vemos en un primer plano detrás de la ventana. Se asoma, pero su rostro queda parcialmente oculto por las cortinas mientras escuchamos su narración:

No creo que sea muy inteligente, pero hay quienes piensan lo contrario. Lo que me falta en la cabeza lo compenso con un exceso de fuerza de voluntad. Soy terca y testaruda, y persigo tenazmente mis objetivos... Básicamente, quería que este complejo fuera mío

La ambición de Marian es, en gran medida, el hilo conductor de la trama: su deseo de evolucionar socialmente es ya evidente en la primera escena de la serie y hay una clara evolución de su situación entre la primera secuencia y el final del quinto episodio. Sin embargo, la caracterización de este protagonista dista mucho de ser sólo positiva. De hecho, Marian está celosa de Raquel (Maribel Verdú), su amiga de la infancia, y las dos mujeres llegarán al punto de enfrentamiento en la segunda parte de la serie, tanto por motivos políticos como personales, ya que se pelean con Martín (Jorge Sanz). Además, la secuencia final comienza con Marian y Martín, ciego y desfigurado en gran parte por su culpa, a quien le dice: "Es cierto que todo podría haber sucedido de otra manera, pero en el fondo no tenemos de qué quejarnos" porque ha conseguido heredar el balneario de acuerdo con el deseo que tenía desde la infancia. Esta frase muestra toda la frialdad y falta de empatía que le permitió cumplir su sueño. En general, el progreso social de Marian no se logra a través de su trabajo o sus habilidades profesionales, sino a través de la manipulación y la seducción, poderes tradicionalmente asociados a una visión estereotipada y negativa de la feminidad.

Con la excepción del caso de Marian, los roles de empleadas domésticas, amas de casa o prostitutas son casi los únicos a los que acceden las mujeres en estas series históricas. De hecho, como muestran Lacalle y Gómez (2016: 63), las mujeres que no realizan ningún tipo de actividad profesional son las más frecuentes en este tipo de ficciones. Además, su importancia para el desarrollo de la trama es casi nula. Esto puede llegar a casos como el de la serie biográfica Miguel Servet (José María Forqué, 1989) donde los personajes femeninos sólo aparecen tras quince minutos de narración en el primer episodio, o treinta y cinco minutos en el cuarto. Además, su media de edad es mucho 
más elevada que en los dramas anclados en la actualidad y, al ser mujeres mayores y jubiladas, su presencia es reducida, con papeles poco definidos.

Otra serie histórica, El olivar de Atocha (Carlos Serrano, 1989), da un lugar algo más importante a los personajes femeninos en su reparto. Estamos hablando de una telenovela que se emitía diariamente entre las 15:35 y las 16:30 horas y en el que la trama romántica y las preocupaciones cotidianas dominan -e incluso dictan- la acción (Downing, 1974: 136). La descripción que hace la página de RTVE de esta serie es en sí misma reveladora:

Crónica costumbrista de una familia liberal republicana, que se remonta a la España del 98 y finaliza días antes de la sublevación militar del 36, cuyos protagonistas son Antonio Malmedina, hombre fundamentalmente bueno que sirve de referencia para analizar la historia de España en una época en que la gente tuvo que definir sus tendencias, Manolita, su temerosa y frágil mujer, y Mariquita, la criada, que es como un miembro más de la familia y la figura cómica de la serie.

El guión se basa en las aventuras que Antonio (Nacho Martínez) tiene que afrontar tras llegar a Madrid a pie desde Málaga, sus encuentros y su evolución profesional como carpintero. Como afirma Jean-Pierre Esquenazi (2017: 39), la presentación de los personajes dice mucho sobre su lugar en la narración. En nuestro caso, Manolita (Enriqueta Carballeira) es descrita como una persona apocopada, falta de autonomía. Sólo aparece en el primer episodio tras 25 minutos de emisión (de un total de 45 ) y sus intervenciones suelen estar ligadas a la acción de un hombre: su hermano en los primeros episodios, y su marido durante la mayor parte de la serie. Sus únicos momentos con otras mujeres son con su madre, antes de casarse con Antonio, y sus conversaciones con Mariquita (Amparo Soler Leal). Vemos así como se establecen relaciones de género en estas ficciones que pasan por la invisibilización de las mujeres.

\subsection{Trabajo doméstico y extracción social}

El personaje de Mariquita nos interesa particularmente en el contexto de nuestra investigación porque, como se desprende de la descripción, las mujeres que trabajan como sirvientas aparecen casi sin excepción como figuras exuberantes, torpes y suavemente insolentes. En general, las mujeres de la clase trabajadora son tradicionalmente retratadas negativamente y caracterizadas por el exceso (Skeggs, 2005: 966) usándose como contrapunto para apoyar la respetabilidad burguesa y blanca (Skeggs, 2004: 99-100). Es un motivo que se repite a lo largo de todas las series visionadas: hablan a gritos, se entromenten en los asuntos de la familia y quieren a todos los miembros de la casa con igual fervor. Parecen totalmente desvinculadas del mundo exterior, hasta el punto de que en ninguna de las series analizadas tienen familiares o amigos fuera de la casa para la que trabajan. Su vida anterior y exterior no existen, salvo 
por otro atributo compartido por todas ellas: no dominan el idioma "culto" y hablan con un acento pronunciado, generalmente el del sur de España. De hecho, cuando las criadas tienen un papel en la acción, son en su mayoría de origen andaluz. Se trata de un tipo común que sigue presente en la ficción española: las mujeres andaluzas suelen ser personajes secundarios y actúan como contrapunto cómico de los protagonistas. Así lo demuestran María Jesús Ruiz Muñoz e Inmaculada Sánchez Alarcón (2008: 150) cuando analizan los estereotipos andaluces en una de las series más populares de la segunda mitad de los 90:

Una figura que resulta el compendio de todos estos rasgos y cuyo éxito es, además, muy significativo de la pervivencia de esta representación es Juani, la empleada de hogar de la serie televisiva Médico de Familia (1995). Se trata de un personaje definido de la manera más llamativa, de baja extracción social, sin estudios y con un carácter sobreprotector. Evidentemente no es causal que sea la criada andaluza de una de las series de mayor repercusión de los últimos años el personaje en el que se materializan todos estos principios.

La misma observación se puede hacer en una serie como Eva y Adán, agencia matrimonial, que no es una serie histórica, sino que su trama se desarrolla en la época contemporánea. La tía de la protagonista y su amiga Ramona encajan perfectamente en el tipo de personaje que acabamos de describir (fig. 1). La tía se nos presenta en el primer episodio, y a pesar de su aparición en otros dos episodios de la serie, el público ni siquiera conoce su nombre, lo que delata su escasa

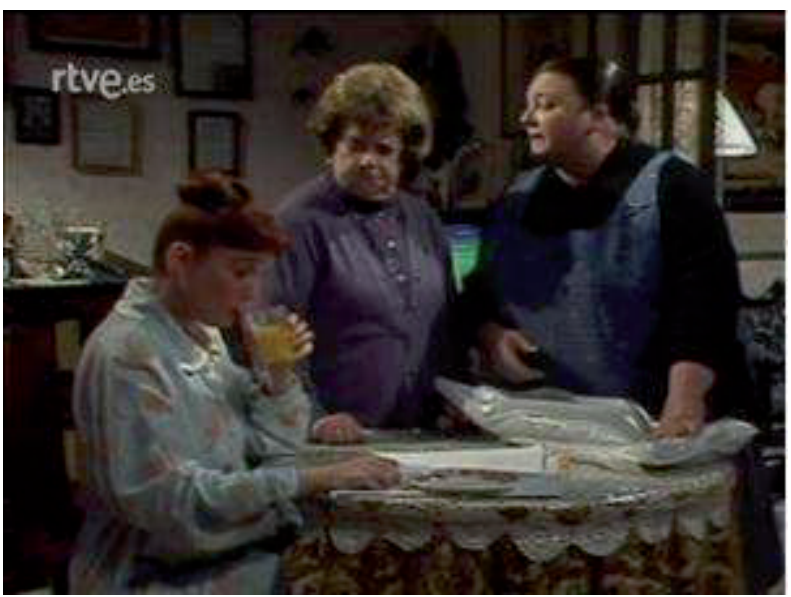

Figura 1. La tía de Eva y su vecina Ramona en la secuencia analizada importancia en la narración. En la secuencia está en casa cuando Eva trabaja en sus notas, la televisión está encendida de fondo con un partido de fútbol y Ramona, la vecina, entra en el salón. La escena busca producir efectos cómicos a partir de los malentendidos entre Eva y su tía sobre los problemas de salud del hijo de Bruno (Antonio Resines), otro protagonista de la serie. El niño sufre de enuresis. La tía, por supuesto, no conoce el término y lo confunde primero con la neurosis y luego con el aneurisma, otra enfermedad que tenía el personaje de una película que habían visto hacía poco. La falta de cultura y prácticas lingüísticas distintas al castellano hablado por las clases medias son por tanto características recurrentes entre los personajes de extracción popular y reflejo de una insuficiente educación (Dalibert, 2018: 8). 
Encontramos una excepción a este estereotipo en la serie Una hija más (Belén Molinero y Lorenzo Zaragoza, 1991), que en este punto es adelantada a su tiempo. La criada, Nati, aparece como una mujer joven, moderna y abierta. Sin embargo, comparte algunos de los rasgos mencionados anteriormente, como la devoción a la familia, la ausencia de vida fuera del hogar, y otro que aún no hemos hecho referencia: está absolutamente desexualizada. De hecho, las criadas rara vez son objeto de seducción por parte de los otros protagonistas de nuestras series. Como ocurre en ésta, donde gran parte de la trama y de las situaciones cómicas giran en torno a la aparente voracidad sexual de Demetrio (Miguel Rellán), el padre de familia que acoge a Amanda, una joven estudiante norteamericana. Vemos como las mujeres del personal doméstico no son pues una amenaza para la familia tradicional.

Ya sean sirvientas o amas de casa, no es habitual mostrar a estos personajes femeninos - siempre secundarios - como inteligentes, autosuficientes y con un buen dominio de la lengua. Desde el punto de vista emocional, encuentran la felicidad en la familia, en la suya o en la que trabajan. Estas mujeres deben ser capaces de comprender los problemas de todos los miembros de la familia, de todas las generaciones del hogar, y proporcionar un apoyo continuo a sus maridos, cuyas preocupaciones acogen $y$ comparten. Cuando tienen aventuras con hombres que no son sus maridos, siempre acaban sufriendo porque son "buenas" esposas (Downing, 1974: 16). Como hemos visto, su mundo se limita al hogar y su socialización se restringe a los miembros de la familia y el valor que crean suele carecer de reconocimiento económico y social (Federici, 2012: 16-17).

\subsection{Mujeres de la posmodernidad}

Es interesante descubrir que, en las series ambientadas en la época contemporánea, las jóvenes protagonistas de nuestro corpus nunca se dedican a las tareas domésticas, ni se nos muestran las trabajadoras domésticas que podrían realizarlas. Sin embargo, nos parece esencial señalar que el trabajo doméstico no desaparece, aunque se haga invisible. En una sociedad postindustrial de servicios, el trabajo doméstico sigue estando muy presente y no puede mecanizarse por completo: sigue requiriendo una cantidad importante de trabajo humano (Federici, 2012: 107). Y, sin embargo, en el segundo plano se obvia a los trabajadores del espacio público o doméstico: es bastante raro ver a conductores de autobús, repartidores, vendedores, personal de limpieza... Esta ausencia puede deberse a las limitaciones económicas de la serie, que busca reducir al máximo el reparto (Downing, 1974: 132), pero nos parece que también responde a opciones narrativas que tienden a minimizar la presencia de las clases trabajadoras.

Siguiendo a Beverley Skeggs (2004: 99), defendemos la idea de que a ciertas representaciones se les asigna un valor, tanto moral (bueno/malo) como económico 
(valioso/no valioso). Del estudio de los personajes de las series de nuestro corpus se desprende que la profesión es un factor importante en la atribución de este valor. Esto explicaría por qué la mayoría de las protagonistas femeninas de las series contemporáneas -que siguen las directrices del PIOM que promueven nuevas representaciones sociales de la mujer - son mujeres trabajadoras. Sin embargo, esta elección de personajes femeninos activos va acompañada de una selección por parte de productores y guionistas de figuras femeninas solas de entre veinte y treinta años para todas las series ambientadas en el presente (de la época). Esta orientación narrativa y estética obedece a la sexualización del cuerpo femenino en la pantalla $-y$, por tanto, a la mirada masculina -, pero sólo se corresponde a medias con la realidad profesional de las mujeres que, entre 1989 y 1993, tenían una tasa de empleo entre el 35,34 y el 38,35 en el rango de edad de 20 a 54 años (Rodriguez Osuna, 1997: 121). Es el caso de Brigada Central, serie policial ambientada en una comisaría de Madrid, donde la única mujer casada, Julia (Assumpta Serna), es la pareja del personaje central, Flores el gitano (Imanol Arias). Las otras protagonistas femeninas trabajan en la comisaría: Carmela y Virgina (Isabel Serrano, Ana Duato) como policías y Rosi (Emma Ozores) como secretaria del comisario. Dado que la edad es un valor de género - sujeto a una doble moral desfavorable para las mujeres en comparación con los hombres (Sontag, 1972)-, las mujeres son más jóvenes que los hombres, y sus personajes rara vez progresan en su carrera profesional, a diferencia de Flores, a quien vemos evolucionar a lo largo de la serie.

En esta unidad especial de investigación que rastrea las principales redes de delincuencia (tráfico de drogas, terrorismo, prostitución, etc.), se expone un mundo masculinizado, característico del género. Las mujeres policías -que aquí están sobrerrepresentadas en relación a su presencia real en el cuerpo policial (Lacalle y Gómez, 2016: 65) - deben adquirir los atributos de sus compañeros para realizar su trabajo. Esto es particularmente evidente en una de las primeras intervenciones de Carmela, cuando se infiltra en un grupo de camellos y es capaz de dominar y detener a dos de estos hombres sin ayuda. Estas mujeres son fuertes, decididas y toman la iniciativa, pero, a diferencia de sus colegas masculinos, no tienen una relación de camaradería entre ellas, sino todo lo contrario: en la mayoría de los episodios hay secuencias, normalmente bastante breves, en las que la rivalidad entre Carmela y Virginia es evidente. Mientras que sus colegas masculinos bromean juntos, se abrazan y se van de copas, ellas no consiguen entablar una verdadera amistad femenina. Están por tanto en constante competencia, tanto en el ámbito profesional como en su capacidad de seducción.

Esta es otra de las características de los personajes femeninos mostrados en su lugar de trabajo en las ficciones analizadas: al igual que Virginia, Carmela o Rosi en nuestra serie policial, la contextualización de su esfera privada es casi inexistente, un rasgo, además, especialmente extendido en este género ficcional (Lacalle y Gómez, 2016: 64). Sólo se 
relacionan dentro de la comisaría, no tienen amistades fuera del trabajo, y cuando tienen historias románticas, siempre es con sus compañeros. La comparación con sus homólogos masculinos vuelve a ser significativa aquí porque, desde el primer episodio, se nos presenta la familia del protagonista, Flores, pero también las relaciones amorosas del agente Lucas (José Coronado) o las relaciones aportando un tono cómico del comisario Poveda (José Manuel Cervina) con sus hijos y, sobre todo, con su mujer. De hecho, el comisario contribuye de dos maneras distintas al espíritu de esta serie, por un lado, es la figura de autoridad, el comisario capaz de gestionar los casos con racionalidad y diligencia, pero por otro lado, su carácter autoritario provoca parte de las situaciones cómicas de este thriller: no puede controlar su ira y se pasa el tiempo gritando a su mujer y a su secretaria Rosi. Como analizan Charo Lacalle y Beatriz Gómez (2016: 62-63), Rosi cumple con los atributos asignados a las mujeres en roles administrativos: son atractivas, serviles y casi sin problemas de relación. En efecto, Rosi siempre está sonriendo, incluso cuando Poveda le grita o la agarra del brazo de forma violenta; siempre va vestida con faldas y/o vestidos cortos que muestran su figura; además, es el único personaje femenino que siempre aparece maquillado, listo para ser consumido por la mirada masculina, según el patrón del texto clásico de Laura Mulvey (fig. 2).

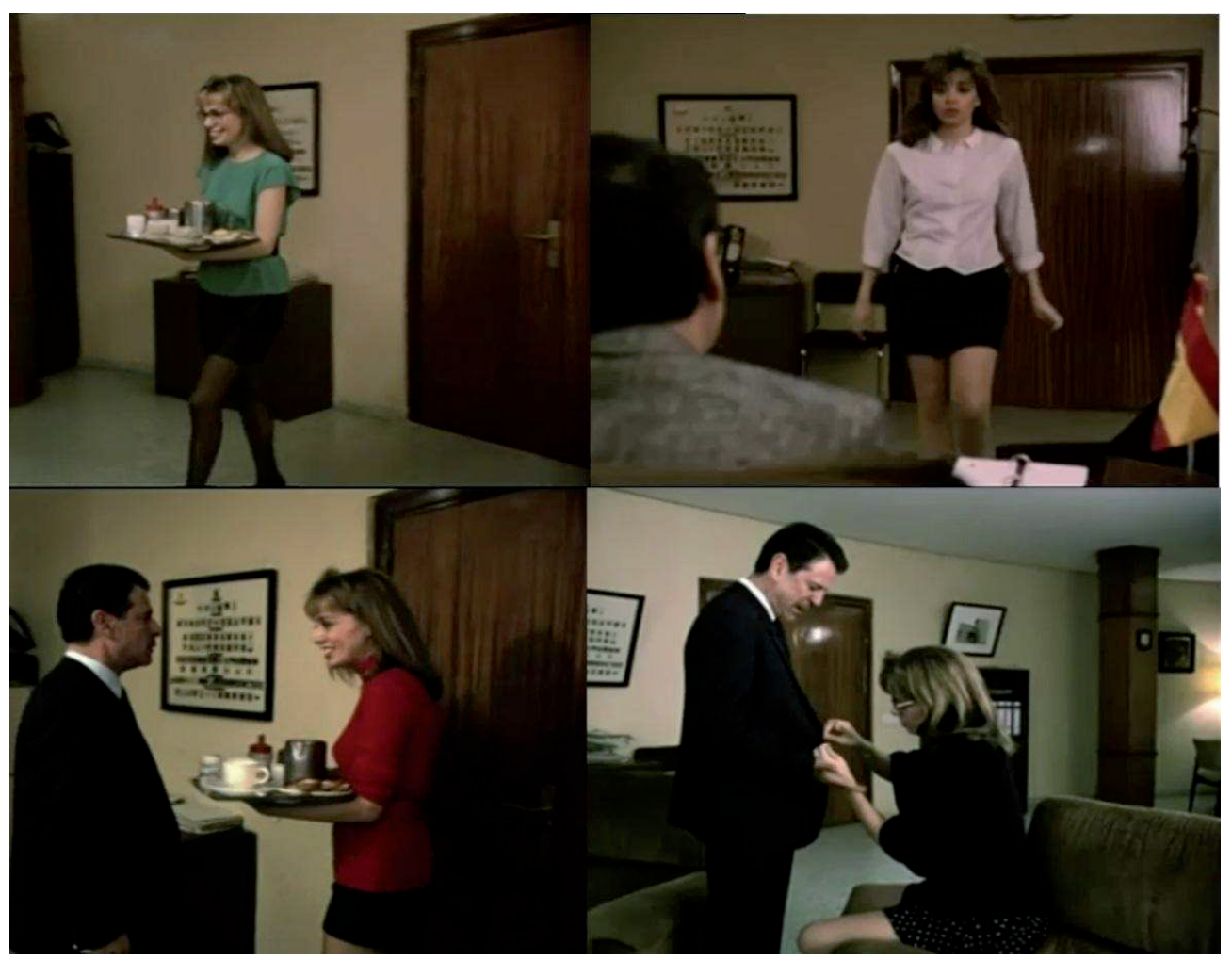

Figura 2. Apariciones de Rosi en los episodios 1, 2, 3 y 5.

La mirada masculina es aún más evidente en series como La mujer de tu vida (Fernando Trueba, 1990) o Delirios de amor (Antoni Capellà y Alberto Espada, 1989), que cuentan historias sentimentales fuera de lo común -eso si, todas heteronormativas-, diferentes en cada episodio, y dirigidas por diferentes directores. El esquema narrativo 
se repite en ambas series, donde generalmente un hombre nos cuenta la historia de su encuentro con una mujer de la que se enamora, y las aventuras que vive para conquistarla, o los fracasos que tiene que soportar. La gran mayoría de las mujeres de estas dos series no trabajan, o al menos su profesión no se menciona en estas tramas. Además, tampoco realizan tareas domésticas. Se nos presentan como mujeres independientes, libres de sus elecciones - elecciones que casi siempre se limitan al ámbito sentimental - , bellas y atractivas, con conciencia de su poder de atracción sobre los hombres. De hecho, parece que su única ocupación es complacer a los hombres, a menudo de forma asumida. Estas protagonistas se definen a sí mismas como objetos sexuales, a los ojos de los hombres pero también para ellas mismas (McRobbie, 2009: 101-102) y de esta manera refuerzan el doble consumo al que nos referimos anteriormente, que está directamente relacionado con las afirmaciones de Mulvey: las espectadoras quedan así encerradas en una mirada masculina al consumir la imagen femenina que transmiten estas ficciones, al tiempo que son orientadas hacia el consumo de moda y productos de belleza (Fig. 3).

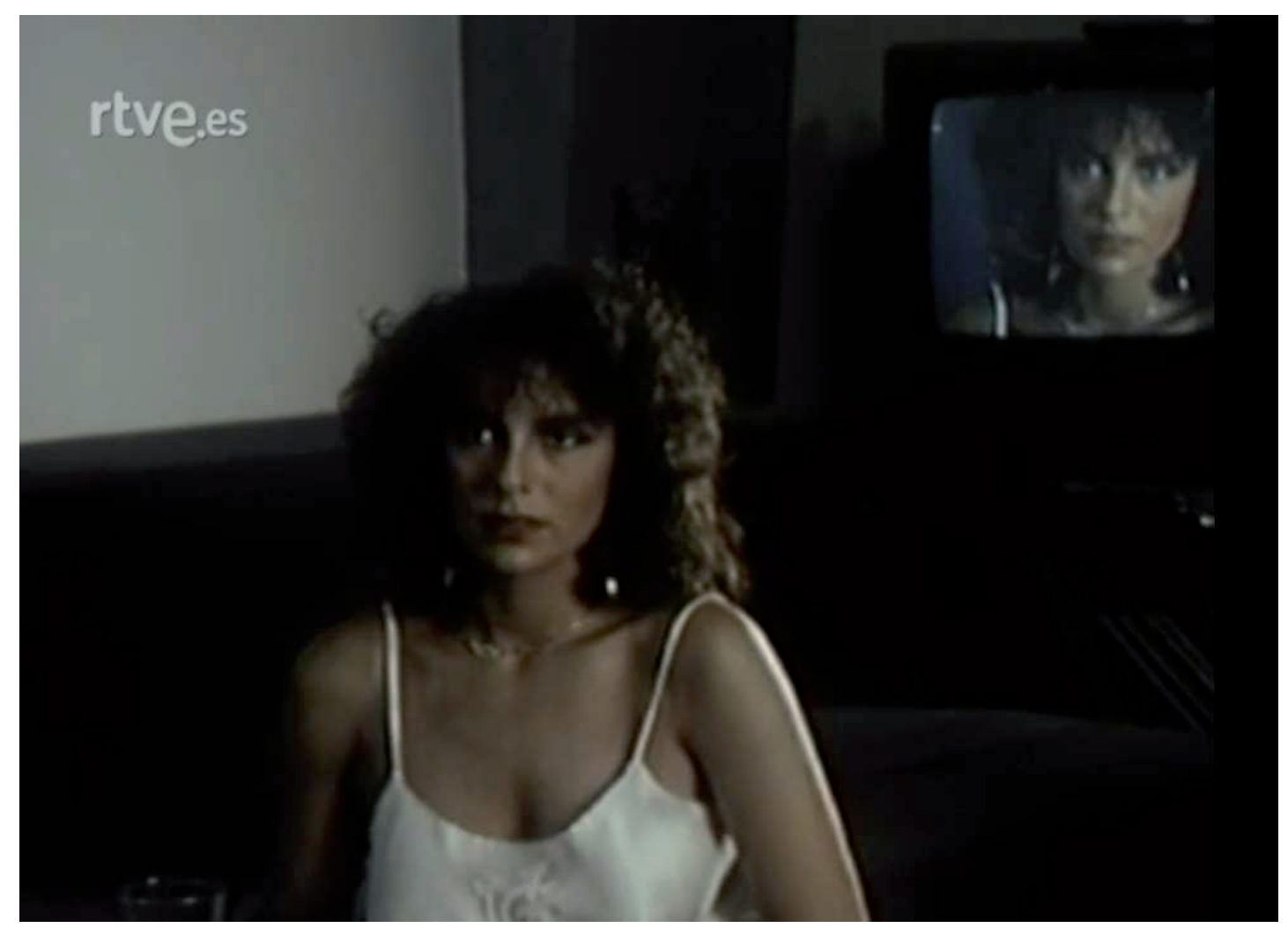

Figura 3. Protagonista femenina del segundo episodio de Delirios de amor prisionera de la mirada del hombre que le filma. 


\section{Conclusión}

La creación de un imaginario social ampliamente despolitizado, de un modelo de sociedad en el que la lucha de clases queda enterrada en favor de la modernización postindustrial, es característica del espíritu de consenso y del ascenso definitivo de las lógicas tecnocráticas y neoliberales durante los años ochenta. Las iniciativas políticas del gobierno socialista y del Instituto de la Mujer a finales de los años 80 trataron de promover la igualdad de oportunidades entre hombres y mujeres: las recomendaciones hechas a los medios de comunicación estatales en el PIOM y la Ley General de Publicidad van en esta dirección. Nuestro análisis se ha centrado en las series como producto clave de la televisión pública, en un momento de creciente competencia con la aparición de las cadenas privadas, basadas en la búsqueda de altas audiencias, "sin importar cómo" (Duran-Froix, 2009: 1-5). Las audiencias a las que se llega son mayoritariamente de clase trabajadora, público privilegiado de este medio.

Como productos culturales, las series formulan discursos sobre la realidad, que no siempre son coherentes y objetivables (Burch \& Sellier, 2009, pág. 10). Hemos igualmente enumerado algunos de los actores que participan en el proceso de creación y programación de estas ficciones. No podemos afirmar que existan modelos femeninos cerrados y unívocos transmitidos por estas producciones, pero sí es posible, a través del análisis de todas las series producidas por la cadena pública durante este periodo concreto, extraer motivos recurrentes en la forma de representar a las mujeres. Así, hemos mostrado cómo las mujeres de la clase trabajadora eran sistemáticamente relegadas a un segundo plano, o incluso invisibilizadas.

De todos los personajes principales estudiados, sólo una mujer -Marian en Los jinetes del Alba- pertenece a las clases populares. En las otras series analizadas, las mujeres de clase trabajadora tienen papeles secundarios, a menudo de poco valor, o incluso sirven de contrapunto cómico. Estas representaciones responden a opciones narrativas, pero también a modelos sociales que excluyen las clases trabajadoras y hacen que sean ignoradas tanto en la ficción como en las políticas públicas. Así, estos años vieron, por ejemplo, la organización y coordinación de asociaciones locales y provinciales de trabajadoras domésticas en respuesta a un decreto de 1985, en el que el gobierno socialista incluía el trabajo doméstico en el estatuto de los trabajadores en condiciones subalternas. Sin embargo, a pesar de estas movilizaciones, los poderes públicos nunca han prestado atención a las condiciones laborales de esta profesión. Esta desconsideración proviene de una imagen que se transmitió a finales de la década y que se agravó con el giro socioliberal del gobierno de Felipe González, y posteriormente con el liberalismo desacomplejado de José María Aznar. En general, las clases trabajadoras siguen siendo caricaturizadas en las producciones audiovisuales a través de figuras estereotipadas como la choni (Oliva, 2014). Este último ejemplo nos muestra también la importancia de este tipo de representaciones en la afirmación de una clase media 
respetable y moderna, que se desmarca de las mujeres de clase trabajadora relegadas al rango de "abyectos de la nación" (Skeggs, 2004: 23).

Cuando los personajes femeninos desempeñan un papel importante en las series de nuestro corpus, son sistemáticamente jóvenes, activas, urbanas y forman parte de una determinada visión de la modernidad. Pertenecen a una clase social en la que, inconscientemente, se invita a los espectadores a proyectarse. Sin embargo, esta proyección se encierra en una mirada masculina hegemónica que hace de estas jóvenes un objeto de consumo, a la vez libidinoso y que responde a la lógica puramente comercial en la que se basa la televisión: su ropa y accesorios, su maquillaje, remiten a los espectadores a los mercados de consumo (McRobbie, 2009: 58-59).

Al destacar a personajes urbanos que gozan de estabilidad profesional y sin limitaciones económicas aparentes, las series difunden un modelo de sociedad en el que los logros sociales están vinculados al mérito y no al origen (nacional, social, racial). Este discurso, que proviene de la revolución neoconservadora y se integra en las políticas socialdemócratas, es posterior al período de los Treinta Años Gloriosos. La generación que se benefició de las condiciones favorables de este periodo se aferra así a esta idea de meritocracia, en la que se apoya para legitimar su propia posición social.

Sin embargo, las promesas de movilidad ascendente y desarrollo económico características del liberalismo de los años 90 sólo pudieron cumplirse para una minoría de la población. En este artículo hemos demostrado que la sociedad de los medios de comunicación de masas y las diversas fábricas de sueños apartan a los consumidores de las condiciones de su vida real, a riesgo de generar frustraciones. Además, al ocultar la abrumadora carga de trabajo de las mujeres de la clase trabajadora, la televisión contribuye a su invisibilización. Pero esta invisibilización (estudiada aquí desde la perspectiva de la serie) no sólo se observa en la ficción: es vivida a diario por las mujeres trabajadoras, que se ven constantemente obligadas a reafirmar su existencia ante una sociedad supuestamente meritocrática.

\section{Referencias bibliográficas}

AAVV. (1987): Plan para la Igualdad de Oportunidades de las Mujeres. 1988-1990. Madrid, Ministerio de Educación y Ciencia.

- AAVV. (2004). La identidad de género en la imagen televisiva, nำ6. Madrid: Instituto de la Mujer.

ARRANZ, F. (2010): Cine y género en España. Una investigación empírica. Madrid, Cátedra. 
BARTHES, S. (2011): "Production et programmation des séries télévisées". En SÉPULCHRE, S. (Ed.) : Décoder les séries télévisées. Bruselas, De Boeck, pp. 47-73.

BURCH, N., \& SELLIER, G. (2009): Le Cinéma au prisme des rapports de sexe. Paris, Vrin.

CHALVON-DEMERSAY, S. (2015) "Pour une responsabilité politique des héros des séries télévisée". En Quaderni, 88, pp. 35-51

DALIBERT, M. (2018): "En finir avec Eddy Bellegueule dans les médias. Entre homonationalisme et ethnicisation des classes populaires". En Questions de communication, 33, 2018, pp. 89-109

DE LAURETIS, T. (1984): Alice doesn't. Feminism, Semiotics, Cinema. Bloomington, Indiana University Press.

DOWNING, M. (1974): "Heroine of the Daytime Serial". En Journal of Communication, 24(2), pp. 130-137.

DURAN-FROIX, J.-S. (2009): La télévision espagnole. Paris, Ophrys.

FEDERICI, S. (2012): Revolution at Point Zero. Housework. Oakland, PM Press.

FRASER, N. (2016): “Contradictions of Capital and Care”. New Left Review, 100, pp. 99117.

GIDDENS, A. (1998): The Third Way. Cambridge, Polity Press.

ESKENAZI, J.-P. (2017) : Élements pour l'analyse des séries. Paris, L'Harmattan.

JURADO, J. (2021). Economía del cine franquista. Protección, corrupción y legitimación de la dictadura. Granada, Comares.

-(2017). El patriotismo constitucional en las series de la TVE durante el primer gobierno socialista. En E. d. Colmenares, Nuevas narrativas. Entre la ficción y la información: de la desregulación a la integración transmedia. Barcelona, Universitat Autònoma de Barcelona, pp. 497-506.

LACALLE, C., \& GÓMEZ, B. (2016): “La representación de las mujeres trabajadoras en la ficción televisiva española". En Comunicar, 24(47), pp. 59-67.

MACÉ, E. (2000): “Qu'est-ce qu'une sociologie de la télévision ? Esquisse d'une théorie des rapports sociaux-médiatisés". En Réseaux, 18(104), pp. 245-288.

McROBBIE, A. (2009): The Aftermath of feminism. Gender, culture and Social Change. Londres, Sage. 
MORENO DÍAZ, J., \& MEDINA, E. (2017): "La producción de concursos en las cadenas de TV españolas : la espectacularización del formato (1990-2000)". En Comunicación y Medios, 35, pp. 64-79.

OLIVA, M. (2014): “Celebrity, Class and Gender in Spain: an Analysis of Belén Esteban's image". En Celebrity Studies, 5(4), pp. 438-454.

QUAGgIO, G. (2013): "La cultura como Prometeo colectivo. Apuntes sobre la política cultural de Javier Solana”. En SOTO CARMONA, A. \& MATEO LÓPEZ, A.: Historia de la época socialista. España 1982-1996. Madrid, Silex, pp. 455-482.

RILEY, S., EVANS, A., \& MACKIEWICZ, A. (2016): "It's just between girls: Negotiating the postfeminist gaze in women's 'looking talk'". En Feminism \& Psychology, 26(I), pp. 94-113.

RODRÍGUEZ LÓPEZ, E. (2015): Por qué fracasó la democracia en España. Madrid, Traficantes de Sueños.

RODRIGUEZ OSUNA, J. (1997): “Evolución de la población activa, ocupación y paro en España 1976-1996". En Política y Sociedad, 26, pp. 113-124.

RUIDO, M. (2007): Just do it! Bodies and Images of Women in the New Division of Labour. Recuperado el 27 de 01 de 2020, de http://goo.gl/rFJHgK

RUIZ MUÑOZ, M. J., \& SÁNCHEZ ALARCÓN, I. (2008): La imagen de la mujer andaluza en el cine español. Sevilla, Centro de Estudios Andaluces.

SAUTE-REQUIN. (2019) : "La sérialité aux bords de la fiction télévisée, ou l'intermédialité comme mise en crise du "tout narratif" sériel". En TV/Séries, 15, pp. 1-22.

SKEGGS, B. (2004): Class, Self, Culture. Londres, Routledge.

- (2005). "The Making of Class and Gender Through Visualizing Moral Subject Formation". Sociology, 39(5), pp. 965-982.

SONTAG, S. (23 de septiembre de 1972): "The Double Standart of Aging" . Saturday Review of Society, pp. 29-38.

VALIENTE, C. (1994): El feminismo de estado en España: el Instituto de la mujer. Madrid, Instituto Juan March.

WALBY, S. (2005): "Gender Mainstreaming : Productive Tensions in Theory and Practice". En Social Politics : International Studies ein Gender, State \& Society, 12(3), pp. 321-343.

WILLIAMS, R. (1990). Television, Technology and Cultural Form. Londres, Routledge. 